\title{
A Model for Hepatitis B with Chronological and Infection Ages
}

\author{
T Y Kouakep ${ }^{1 *}$, A Ducroty² and Houpa D D E
}

${ }^{1}$ Ngaoundere University, PO Box 454 Ndang, Ngaoundere, Cameroon

${ }^{2}$ Universite Bordeaux Segalen, UFR Sciences et Modelisation, 3 ter place de la Victoire, Bat. D, 2eme etage, 33076 Bordeaux Cedex, France

${ }^{3}$ Ngaoundere University, PO Box 454 Ndang, Ngaoundere, Cameroon

\begin{abstract}
We construct and study a differential infectivity model with chronological and infection age. The application is done on hepatitis $B$ in Cameroon. We prove the global stability of the disease free equilibrium when the basic reproduction ratio $R_{0}$ is less than one and the existence and uniqueness of an endemic equilibrium when $R_{0}>1$.
\end{abstract}

Keywords: Nonlinear dynamical systems; Global stability; Lyapunov methods; Differential infectivity models; HBV; Cameroon

\section{A Simplified Model without Vertical Transmission and With Control Measure}

Hepatitis B is endemic in Africa [1-3] (see also the references cited therein) and some models have been constructed in order to understand it's dynamic with ODE deterministic (with delay or not) or stochastic processes $[2,4-8]$ or partial differential equations $[9,10]$.

Studies like [9-13] recognized the importance of the age factor in the dynamics of infectious diseases like hepatitis B [10]. Moreover some studies like $[4,14]$ obtained results with ODEs with discrete age(s) that could be generalized with continuous age assumption more realistic and relevant.

We introduce then a model with differential infectivity and chronological or infection ages. We denote by $s(t, a)$ the density of susceptible at time $t$ with chronological aged a. We denote by $i(t, \tau)$ the density of infective that will develop acute disease at time t contaminated since a time and e $(t, \tau)$ the density of infective that will not develop acute disease (asymptomatic carrier) at time t contaminated since a time $\tau$. The model we shall consider reads as follows:

$$
\begin{aligned}
& \left(\partial_{t}+\partial_{a}\right) s(t, a)=-\mu s(t, a)-\lambda(t) s(t, a), \quad t>0, a>0, \\
& s(t, 0)=\Lambda, \\
& \left(\partial_{t}+\partial_{\tau}\right) i(t, \tau)=-\left(\mu+\gamma_{i}\right) i(t, \tau), t>0, \tau>0, \\
& \left(\partial_{t}+\partial_{\tau}\right) e(t, \tau)=-\left(\mu+\gamma_{e}\right) e(t, \tau), t>0, \tau>0, \\
& i(t, 0)=\lambda(t) \int_{0}^{\infty} p(a) s(t, a) d a, e(t, 0)=\lambda(t) \int_{0}^{\infty}(1-p(a)) s(t, a) d a
\end{aligned}
$$

Here $\Lambda>0$ is some constant entering in flux, $\mu>0$ is the natural death rate, $\gamma_{i}$ is the additional death rate due to the disease, $a \rightarrow p(a)$ $\in[0,1]$ is the proportion of individuals going to the acute infective class while $1-p(a)$ is the proportion to not develop the acute disease when infection occurs. Finally, it remains to model $\lambda(t, a)$, the force of infection, those general form can be written in the form

$$
\lambda(t)=\int_{0}^{\infty}\left(\beta_{i}(\tau) i(t, \tau)+\beta_{e}(\tau) e(t, \tau)\right) d \tau
$$

Finally this model is supplemented together with some initial data

$$
\begin{aligned}
& s(0, .)=s_{0}(.) \in L_{+}^{1}(0, \infty) \\
& i(0, .)=i_{0}(.), e(o, .)=e_{0}(.) \text { with }\left(i_{0}, e_{0}\right) \in L_{+}^{1}(0, \infty)^{2}
\end{aligned}
$$

The above model takes into account the chronological age of susceptible. This parameter has strong implication in the dynamics of infection. Indeed, depending on the age at which susceptible enters the infective's classes, the disease will develop indifferent way. For hepatitis $B$ virus (HBV), young infections lead to chronic infection while older infection leads to acute disease.

In the above model, we do not take into account possible vertical transmission and we do not consider any control strategy such vaccination campaign. It seems to be relevant together the assumption of WHO [3] that consider that vertical transmission of the disease does occur in sub-saharian Africa, but its influence of the dynamics of the disease is rather small because the proportion of chronic infections acquired prenatally is low. Under the above assumption, we assume that the chronological age for the infective classes do not play an important role. But the time since infection is a relevant biological variable because of the possibility to have a long latent period (especially for the asymptomatic carrier class, until several years).

The work is organized as follows. In Section 2, we prove the wellposedness of the PDE (1.1-1.2), derive preliminary results useful to study the long term behavior of the model. Sections 3,4 , and 5 is devoted to the uniqueness of endemic equilibrium when the biological basic reproduction rate $R_{0}$ is greater than 1 and study the global asymptotically stability of the disease free equilibrium if $1>R_{0}$. Finally Section 6 presents discussion.

\section{Abstract Cauchy Problem Reformulation}

\section{Mathematical assumptions}

We assume that:

a) $\gamma_{i}(\tau) \equiv \gamma_{i}>0, \gamma_{e}(\tau) \equiv 0$

b) The function $p \in L^{\infty}(0, \infty)$ with $p(a) \in[0,1]$ a.e. and not identically 0 and 1 .

c) Function $\beta_{i}, \beta_{e} \in L_{0}^{\infty}(0, \infty)$.

${ }^{*}$ Corresponding author: T Y Kouakep, Ngaoundere University, PO Box 454 Ndang, Ngaoundere, Cameroon, Tel: 237222540 40; E-mail: kouakep@aims-senegal.org

Received July 26, 2013; Accepted December 04, 2013; Published December 23, 2013

Citation: Kouakep TY, Ducroty A, Houpa DDE (2013) A Model for Hepatitis B with Chronological and Infection Ages. J Appl Computat Math 3: 148. doi:10.4172/21689679.1000148

Copyright: (c) 2013 Kouakep TY, et al. This is an open-access article distributed under the terms of the Creative Commons Attribution License, which permits unrestricted use, distribution, and reproduction in any medium, provided the original author and source are credited. 


\section{Abstract Cauchy problem}

The aim of this section is to deal with (1.1). To do so, we consider the Banach spaces

$$
X=\mathbb{R}^{3} \times L^{1}(0, \infty)^{3}, \quad X_{0}=\left\{0_{\mathbb{R}^{3}}\right\} \times L^{1}(0, \infty)^{3},
$$

endowed the norm $\mid\left(\begin{array}{l}\alpha_{3} \\ \psi_{1} \\ \psi_{2} \\ \psi_{3}\end{array}\right) \|_{X}=\sum_{i=1}^{3}\left(\left|\alpha_{i}\right|+\left|\psi_{1}\right| L_{(0, \infty, \mathbb{R})}^{1}\right)$,

as well as the non-densely defined linear operator $A: D(A) \subset X \rightarrow X$ defined by

$$
D(A)=\left\{O_{\mathbb{R}}{ }^{3}\right\} \times W^{1,1}(O, \infty)^{3}
$$

together with

$$
A\left(\begin{array}{c}
0 \\
0 \\
0 \\
\varphi_{1} \\
\varphi_{2} \\
\varphi_{3}
\end{array}\right)=\left(\begin{array}{c}
-\varphi_{1}(0) \\
-\varphi_{2}(0) \\
-\varphi_{3}(0) \\
-\varphi_{1}^{\prime}(a)-\mu \varphi_{1}(a) \\
-\varphi_{2}^{\prime}(\tau)-\left(\mu+\gamma_{i}\right) \varphi_{2}(\tau) \\
-\varphi_{3}^{\prime}(\tau)-\left(\mu+\gamma_{e}\right) \varphi_{3}(\tau)
\end{array}\right)
$$

as well as the nonlinear map $\mathrm{F}: \mathrm{X}_{0} \rightarrow \mathrm{X}$ defined by

$$
F\left(\begin{array}{c}
0 \\
0 \\
0 \\
\varphi_{1} \\
\varphi_{2} \\
\varphi_{3}
\end{array}\right)=\left(\begin{array}{c}
\Lambda \\
\int_{0}^{\infty} p(a) \varphi_{1}(a) d a \int_{0}^{\infty}\left(\beta_{i}(\tau) \varphi_{2}(\tau)+\beta_{e}(\tau) \varphi_{3}(\tau)\right) d \tau \\
\int_{0}^{\infty}(1-p(a)) \varphi_{1}(a) d a \int_{0}^{\infty}\left(\beta_{i}(\tau) \varphi_{2}(\tau)+\beta_{e}(\tau) \varphi_{3}(\tau)\right) d \tau \\
-\int_{0}^{\infty}\left(\beta_{i}(\tau) \varphi_{2}(\tau)+\beta_{e}(\tau) \varphi_{3}(\tau)\right) d \tau
\end{array}\right),
$$

Let us notice that $\overline{D(A)}=X_{0}$

Now by identifying $(\mathrm{s}(\mathrm{t},),. \mathrm{I}(\mathrm{t},),. \mathrm{e}(\mathrm{t},)$.$) in (1.1) together with$ $\mathrm{u}(\mathrm{t})=(0,0,0, \mathrm{~s}(\mathrm{t},),. \mathrm{i}(\mathrm{t},),. \mathrm{e}(\mathrm{t},),) \mathrm{T}$, one obtains that $\mathrm{u}(\mathrm{t})$ satisfies the following abstract Cauchy problem $\frac{d u(t)}{d t}=A u(t)+F(u(t)), t>0, \quad(2.3)$ together with the initial data $u(0)=x=\left(0,0,0, s_{0}, i_{0}, e_{0}\right)^{T} \in X_{0}$.

We also consider the positive cones $X_{+}=\mathbb{R}^{+3} \times L_{+}^{1}(0, \infty)^{3}, X_{0+}=X_{0} \cap X_{+}$

Lemma 2.1: (Hille-Yosida property) Operator A: $D(A) \subset X \rightarrow X$ is a Hille-Yosida operator. More precisely we have $(-\mu, \infty) \subset \rho(A)$ the resolvent set of $\mathrm{A}$ and for each $\lambda>-\mu$, each $\left(\alpha_{1}, \alpha_{2}, \alpha_{3}, \psi_{1}, \psi_{2}, \psi_{3}\right)^{T} \in X$ we have

$$
(\lambda-A)^{-1}\left(\begin{array}{c}
0_{\mathbb{R}^{3}} \\
\varphi_{1} \\
\varphi_{2} \\
\varphi_{3}
\end{array}\right)=\left(\begin{array}{l}
\alpha_{1} \\
\alpha_{2} \\
\alpha_{3} \\
\psi_{1} \\
\psi_{2} \\
\psi_{3}
\end{array}\right)
$$

$$
\begin{aligned}
& \Leftrightarrow \\
& \left\{\begin{array}{c}
\varphi_{1}(a)=e^{-(\lambda+\mu) a} \alpha_{1}+\int_{0}^{a} e^{-(\lambda+\mu)(a-s)} \psi_{1}(s) d s \\
\varphi_{2}(a)=e^{-\left(\lambda+\mu+\gamma_{i}\right) a} \alpha_{2}+\int_{0}^{a} e^{-\left(\lambda+\mu+\gamma_{i}\right)(a-s)} \psi_{2}(s) d s \\
\varphi_{3}(a)=e^{-\left(\lambda+\mu+\gamma_{e}\right) a} \alpha_{3}+\int_{0}^{a} e^{-\left(\lambda+\mu+\gamma_{e}\right)(a-s)} \psi_{3}(s) d s
\end{array}\right.
\end{aligned}
$$

Moreover we have for each $\lambda>-\mu$

$(\lambda-A)^{-1} X_{+} \subset X_{0+}$

Proof: Let $x=\left(\alpha_{1}, \alpha_{2}, \alpha_{3}, \psi_{1}, \psi_{2}, \psi_{3}\right)^{T} \in X$ and $\lambda>-\mu$ be given. Then the equation

$$
(\lambda-A)\left(\begin{array}{c}
0_{\mathbb{R}^{3}} \\
\varphi_{1} \\
\varphi_{2} \\
\varphi_{3}
\end{array}\right)=x,
$$

rewrites as the following system

$$
\left\{\begin{array}{c}
\varphi_{1}(a)^{\prime}=-(\lambda+\mu) \varphi_{1}(a)+\psi_{1}(a) \\
\varphi_{2}(a)^{\prime}=-\left(\lambda+\mu+\gamma_{i}\right) \varphi_{2}(a)+\psi_{2}(a) \\
\varphi_{3}(a)^{\prime}=-\left(\lambda+\mu+\gamma_{e}\right) \varphi_{3}(a)+\psi_{3}(a) \forall a \geq 0 \\
\varphi_{1}(0)=\alpha_{1}, \varphi_{2}(0)=\alpha_{2}, \varphi_{3}(a)=\alpha_{3},
\end{array}\right.
$$

that is

$$
\left\{\begin{array}{c}
\varphi_{1}(a)=e^{-(\lambda+\mu) a} \alpha_{1}+\int_{0}^{a} e^{-(\lambda+\mu)(a-s)} \psi_{1}(s) d s \\
\varphi_{2}(a)=e^{-\left(\lambda+\mu+\gamma_{i}\right) a} \alpha_{2}+\int_{0}^{a} e^{-\left(\lambda+\mu+\gamma_{i}\right)(a-s)} \psi_{2}(s) d s \\
\varphi_{3}(a)=e^{-\left(\lambda+\mu+\gamma_{e}\right) a} \alpha_{3}+\int_{0}^{a} e^{-\left(\lambda+\mu+\gamma_{e}\right)(a-s)} \psi_{3}(s) d s
\end{array}\right.
$$

On the other hand one has

$$
\left\{\begin{array}{l}
\left\|\varphi_{1}\right\| L^{1}(0, \infty) \leq \frac{\left|\alpha_{1}\right|+\left\|\varphi_{1}\right\|_{L(0, \infty)}^{1}}{\lambda+\mu}, \\
\left\|\varphi_{2}\right\| L^{1}(0, \infty) \leq \frac{\left|\alpha_{2}\right|+\left\|\varphi_{2}\right\|_{L(0, \infty)}^{1}}{\lambda+\mu}, \\
\left\|\varphi_{3}\right\| L^{1}(0, \infty) \leq \frac{\left|\alpha_{3}\right|+\left\|\varphi_{3}\right\|_{L(0, \infty)}^{1}}{\lambda+\mu}
\end{array}\right.
$$

As a consequence,

$(-\mu, \infty) \subset p(A)$ and $\left\|(\lambda-A)^{-1}\right\| L(X) \leq \frac{1}{\lambda+\mu}, \forall \lambda>-\mu$

This completes the proof of the Hille-Yosida property. Finally the explicit formula of the resolvent operator implies that (2.4) holds true.

Theorem 2.2: There exists a continuous semi flow $\{U(t)\}_{\mathrm{t} \geq 0}$ on $X_{0+}$ into itself such that for each $x \in X_{0+}$, the map $t \rightarrow \mathrm{U}(\mathrm{t}) \mathrm{X}$ is the unique integrated solution of (2.3) with initial data $\mathrm{x}$, namely $t \rightarrow \mathrm{U}(\mathrm{t}) \mathrm{x}$ satisfies

(i) $\int_{0}^{t} U(s) x d s \in D(A), \forall t \geq 0$

(ii) $U(t) x=x+A \int_{0}^{t} U(s) x d s+\int_{0}^{t} F(U(s) x) d s$ for each $t \geq 0$ 
Moreover we have for each $x \in X_{0+}$ :

$$
\frac{\Lambda}{\mu+\gamma_{i}+\gamma_{e}} \leq \liminf _{t \rightarrow \infty}\|U(t) x\| x \leq \limsup _{t \rightarrow \infty}\|U(t) x\| x \leq \frac{\Lambda}{\mu}
$$
that

Proof: Let us first notice that for each $M>0$ there exists $\lambda>0$ such

$$
F(x)+\lambda x \in X_{+}, \quad \forall x \in B_{x}(0, M) \cap X_{0+}
$$

With $B_{X}(0, M)$ denote the ball of radius $M$ centered at 0 . One obtains the existence of a maximal positive semi flow for (2.3) on $X_{0+}$ into itself. It remains to prove that this semi flow is globally defined. To do so, let $x \in X_{0+}$ be given and recall that $U(t) x=(0,0,0, s(t, .), i(t, .), e(t, .))^{T}$

Consider the quantity

$$
P(t)=\|U(t) x\| x=\int_{0}^{\infty} s(t, a) d a+\int_{0}^{\infty} i(t, \tau) d \tau+\int_{0}^{\infty} e(t, \tau) d \tau,
$$

the total population at time $t$. Then it satisfies the differential inequality

$$
\frac{d P(t)}{d t} \leq \wedge-\mu P(t)
$$

Thus the map $t \rightarrow P(t)$ cannot blow up in finite and the global existence result follows.

Let us, in addition, notice that, from this inequality one gets (by density)

$$
\limsup _{t \rightarrow \infty}\|U(t) x\| x \leq \frac{\Lambda}{\mu}, \forall x \in X_{0+}
$$

One the other hand one has

$$
\frac{d P(t)}{d t}=\Lambda-\mu P(t)-\left(\gamma_{i} \int_{0}^{\infty} i(t, \tau) d \tau+\gamma_{e} \int_{0}^{\infty} e(t, \tau) d \tau\right) \geq \wedge-\left(\mu+\gamma_{i}+\gamma_{e}\right) P(t)
$$

so that $\liminf _{x \rightarrow \infty}\|U(t) x\| x \geq \frac{\Lambda}{\mu+\gamma_{i}+\gamma_{e}}, \forall x \in X_{0+}$

This completes the proof of the result.

\section{Stationary States}

\section{The disease free equilibrium}

The disease free equilibrium corresponds to a stationary (that is time independent solution)

$$
\left(s_{F} \equiv s_{F}(a), i_{F} \equiv 0, e_{F} \equiv 0\right),
$$

of (1.1)-(1.2). As a consequence we have the following lemma

Lemma 3.1: The dynamical system provided by (1.1)-(1.2) has a unique disease free equilibrium where $\mathrm{sF}$ is given by $s(a), \equiv \wedge e$

From now one we set

$$
S_{1}=\int_{0}^{\infty} p(a) s_{F}(a) d a, \quad S_{2}=\int_{0}^{\infty} p(a) s_{F}(a) d a,
$$

and we consider the biological basic reproduction rate

$$
R_{0}=\Lambda \int_{0}^{\infty}\left(P_{i} \beta_{i}(\tau) e^{-v_{i} \tau}+P_{e} \beta_{e}(\tau) e^{-v_{e} \tau}\right) d_{\tau}
$$

where we have set

\section{Endemic equilibrium}

We look for stationary solutions (s; i; e) such that (i; e) not identically zero satisfying

$$
\begin{aligned}
& s^{\prime}(a)=-\mu s(t, a)-\lambda s(a), a>0, \\
& s(0)=\Lambda, \\
& i^{\prime}(\tau)=-\left(\mu+\gamma_{i}\right) i(\tau), \tau>0, \\
& e^{\prime}(\tau)=-\left(\mu+\gamma_{e}\right) e(\tau), \tau>0, \\
& i(0)=\lambda \int_{0}^{\infty} p(a) s(a) d a, e(0)=\lambda \int_{0}^{\infty}(1-p(a)) s(a) d a \\
& \lambda \int_{0}^{\infty}\left(\beta_{i}(\tau) i(\tau)+\beta_{e}(\tau) e(\tau)\right) d \tau,
\end{aligned}
$$

Theorem 3.2: Recalling definition (3.5), if $R_{0}>1$, system (1.1)-(1.2) has a unique endemic equilibrium point denoted by $\left(s_{E^{\prime}} ; i_{E^{\prime}} ; e_{E}\right)$.

Proof: we have

$$
\begin{aligned}
& s(a)=\Lambda e^{-(\mu+\lambda)}, \\
& i(\tau)=e^{-\left(\mu+\lambda_{i}\right) \tau} \lambda \Lambda \int_{0}^{\infty} p(a) e^{-(\mu+\lambda) a} d a \\
& e(\tau)=e^{-\left(\mu+\lambda_{e}\right) \tau} \lambda \Lambda \int_{0}^{\infty}(1-p(a)) e^{-(\mu+\lambda) a} d a
\end{aligned}
$$

Thus

$$
\begin{aligned}
& \lambda=\int_{0}^{\infty} \beta_{i}(\tau) e^{-\left(\mu+\gamma_{i}\right) \tau} d \tau \lambda \Lambda \int_{0}^{\infty} p(a) e^{-(\mu+\lambda) a} d a \\
& +\int_{0}^{\infty} \beta_{e}(\tau) e^{-\left(\mu+\gamma_{e}\right) \tau} d \tau \lambda \Lambda \int_{0}^{\infty}(1-p(a)) e^{-(\mu+\lambda) a} d a
\end{aligned}
$$

We are looking for endemic stationary state, that is $\lambda>0$, so that

$$
\begin{aligned}
& 1=\int_{0}^{\infty} \beta_{i}(\tau) e^{-\left(\mu+\gamma_{i}\right) \tau} d \tau \lambda \Lambda \int_{0}^{\infty} p(a) e^{-(\mu+\lambda) a} d a \\
& +\int_{0}^{\tau} \beta_{e}(\tau) e^{-\left(\mu+\gamma_{e}\right) \tau} d \tau \lambda \Lambda \int_{0}^{\infty}(1-p(a)) e^{-(\mu+\lambda) a} d a
\end{aligned}
$$

Now the map $\lambda \rightarrow \mathrm{f}(\lambda)$ is non increasing with $f$ defined by

$$
\begin{aligned}
& f(\lambda)=\int_{0}^{\infty} \beta_{i}(\tau) e^{-\left(\mu+\gamma_{i}\right) \tau} d \tau \lambda \Lambda \int_{0}^{\infty} p(a) e^{-(\mu+\lambda) a} d a \\
& +\int_{0}^{\tau} \beta_{e}(\tau) e^{-\left(\mu+\gamma_{e}\right) \tau} d \tau \lambda \Lambda \int_{0}^{\infty}(1-p(a)) e^{-(\mu+\lambda) a} d a
\end{aligned}
$$

and $f(\lambda) \rightarrow 0$ when $\lambda \rightarrow \infty$. As a consequence, since $R_{0}=f(0)>1$, there exists a unique $\lambda_{\mathrm{E}}>0$ such that

$$
f \lambda_{\mathrm{E}}=1 \text {. }
$$

Finally, the functions

$$
\begin{aligned}
& s_{E}(a)=\Lambda e^{-\left(\mu+\lambda_{E}\right) a}, \\
& i_{E}(\tau)=e^{-\left(\mu+\gamma_{i}\right) \tau} \lambda_{E} \Lambda \int_{0}^{\infty} p(a) e^{-\left(\mu+\lambda_{E}\right) a} d a \\
& e_{E}(\tau)=e^{-(\mu+\gamma e) \tau} \lambda_{E} \Lambda \int_{0}^{\infty}\left(1-p(a) e^{-\left(\mu+\lambda_{E}\right) a} d a\right.
\end{aligned}
$$

provides the unique endemic stationary state of system (1.1)-(1.2).

\section{Dynamical Properties}

\section{Assumption 4.1}

Assume that the maps $a \rightarrow \beta_{\mathrm{i}}(a)$ and $a \rightarrow \beta_{\mathrm{e}}(a)$ are bounded and uniformly continuous from $[0, \infty)$ into itself.

\section{Volterra Integral Formulation:}

The solutions of (1.1)-(1.2) can be reformulated as follows

$$
\partial_{t} s+\partial_{a} s=-\mu s-\lambda(t) s
$$$$
s(t, 0)=\Lambda, s(0, .)=s_{0} \text {, }
$$

With 


$$
\begin{aligned}
& \lambda(t)=\int_{0}^{\tau} \beta_{i}(\tau) i(t, \tau)+\beta_{e}(\tau) e(t, \tau) d \tau, \\
& \text { and } \\
& i(t, \tau)=\left\{\begin{array}{c}
e^{\left(\mu+\gamma_{i}\right) t} i_{0}(a-t) \text { if } a-t \geq 0, \\
e^{\left(\mu+\gamma_{i}\right) a} b_{i}(t-a) \text { if } a-t<0
\end{array}\right\}, \quad e(t, \tau)=\left\{\begin{array}{c}
e^{(\mu+\gamma) t} e_{0}(a-t) \text { if } a-t \geq 0, \\
e^{\left(\mu+\gamma_{e}\right) a} b_{e}(t-a)
\end{array}\right.
\end{aligned}
$$

where $t \rightarrow b_{i}(t)=i(t, 0)$ and $t \rightarrow b_{e}(t)=e(t ; 0)$ are the unique continuous functions satisfying for $k=$ i.e:

$$
\begin{aligned}
& b_{k}(t)=k(t, 0)=p_{k}[s(t, .)] \int_{0}^{t}\left(\beta_{i}(\tau) e^{v_{i} \tau} b_{i}(t-\tau)+\beta_{e}(\tau) e^{v_{e} \tau} b_{e}(t-\tau)\right) d \tau \\
& +p_{k}[s(t, .)] \int_{t}^{\infty}\left(e^{v_{i} t} \beta_{i}(\tau) i_{0}\left(\tau-t+e^{v_{e} t} \beta_{e}(\tau) e_{0}(\tau-t)\right) d \tau\right.
\end{aligned}
$$

where we have set

$$
p_{i}[\varphi]=\int_{0}^{\infty} p(a) \varphi(a) d a, p_{e}[\varphi]=\int_{0}^{\infty}(1-p(a)) \varphi(a) d a .
$$

By using results in Sell and You [15], one can find suitable conditions to prove that $\{U(t)\}_{t \geq 0}$ is asymptotically smooth and derived the following proposition.

Proposition 4.2: Let Assumption 4.1 be satisfied. Then there exists a compact set $A \subset X_{0+}$ such that

(i) A is invariant under the semi flow $\{U(t)\}_{t \geq 0}$

(ii) A attracts the bounded sets of $X_{0+}$ under $\{U(t)\}_{t \geq 0}$. This means that for each bounded set $B \subset X_{0+}$ we have

$$
\lim _{t \rightarrow \infty} \delta(U(t) B, A)=0,
$$

where $\delta$ is defined as

$$
\delta(A, B)=\sup _{x \in A} \inf _{y \in B}\|x-y\|
$$

Moreover A is locally asymptotically stable. Next one considers the following quantities $\tau_{k}=\sup \left\{\tau \geq 0: \beta_{k}(\tau)>0\right\}, k \in\{i, e\}$ and the following set:

$$
\widehat{M}=\left\{(\varphi, \psi) \in L_{+}^{1}(0, \infty)^{2}: \int_{0}^{\tau_{i}} \varphi(\tau) d \tau+\int_{0}^{\tau_{i}} \psi(\tau) d \tau>0\right\}
$$

We set also

$$
\begin{aligned}
& M_{0}=\left\{O_{\mathbb{R}^{3}}\right\} \times L_{+}^{1}(0, \infty) \times \widehat{M} \subset X_{0+}, \\
& \partial M_{0}=X_{0+} \backslash M_{0}
\end{aligned}
$$

From this Volterra integral formulation one obtains the following lemma:

Lemma 4.3: The sets $M_{0}$ and $\partial M_{0}$ are positively invariant under the semi flow $\{U(t)\}_{t \geq 0}$. Moreover if $x \in \partial M_{0}$ then

$$
\begin{gathered}
\lim _{t \rightarrow \infty}\left\|U(t) x-x_{F}\right\|=0 \\
\text { with } x_{F}=\left(0_{\mathbb{R}^{3}}, s_{F}(.)=\Lambda e^{-\mu \cdot}, 0,0\right)^{T}
\end{gathered}
$$

\section{Global Stability of the Disease free Equilibrium when $R_{0}<1$ and Simulations}

\section{Stability with a Lyapunov like function}

Lemma 5.1: Let $t € R$ and $a>0$ be given. For a globally in time solution s, we get the following inequality:

$$
s(t, a) \leq s_{F}(a)
$$

Proof: At first we want to prove that: $s(t, a) \leq s_{F}(a), \forall a>0$. We have on characteristics:

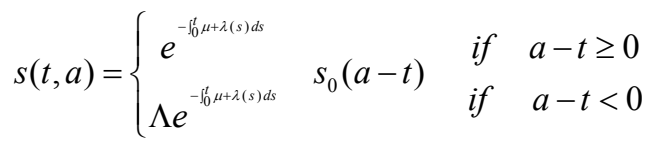

As a consequence we obtain for each $s \in \mathbb{R}, \mathrm{t}>0$ and a $>0$ that

$$
s(t+s, a)=\left\{\begin{array}{c}
e^{-\mu t+f_{0}^{t}+\lambda(s) d s} s(s, a-t) \text { if } a-t \geq 0 \\
\wedge e^{-\mu a-\int_{0}^{t} \lambda+(t)(t) d s} \text { if } \quad a-t<0
\end{array}\right.
$$

If $\mathrm{t}=$ a then for each $s \in \mathbb{R}$ :

$$
s(s+a, a)=e^{-\mu a-\int_{0}^{a} \lambda(s) d s} s(s, 0) \leq S_{F}(a)
$$

Let $t \in \mathbb{R}$ and $\mathrm{a}>0$ be given. Choose $s \in \mathbb{R}$ such that $\mathrm{s}=\mathrm{t}-\mathrm{a}$. The above equality re-writes as

$$
s(t, a) \leq s_{F}(a),
$$

and the result follows.

We will use the lemma above in the proof of the following theorem.

Theorem 5.2: For the model (1.1)-(1.2), if $R_{0}<1$, then the disease free equilibrium is globally asymptotically stable in $\partial M_{0}$.

Proof: Recall that

$$
\begin{aligned}
& \frac{R_{0}}{\wedge}=\int_{0}^{\infty} \beta_{i}(\tau) e^{-\left(\mu+\gamma_{i}\right) \tau} d \tau \int_{0}^{\infty} p(a) e^{-\mu a} d a \\
& +\int_{0}^{\tau} \beta_{e}(\tau) e^{-\left(\mu+\gamma_{e}\right) \tau} d \tau \int_{0}^{\infty}(1-p(a)) e^{-\mu a} d a,
\end{aligned}
$$

Choose and such that

$$
\left\{\begin{array}{c}
\Gamma_{i}^{\prime}(\tau)=\left(\mu+\gamma_{i}\right) \Gamma_{i}(\tau)-\beta_{i}(\tau) \\
\Gamma_{i}(0)=\int_{0}^{\infty} e^{-\left(\mu+\gamma_{i}\right) s} \beta_{i}(s) d s,
\end{array}\right.
$$

And

$$
\left\{\begin{array}{c}
\Gamma_{e}^{\prime}(\tau)=\left(\mu+\gamma_{e}\right) \Gamma_{e}(\tau)-\beta_{e}(\tau) \\
\Gamma_{e}(0)=\int_{0}^{\infty} e^{-\left(\mu+\gamma_{e}\right) s} \beta_{e}(s) d s,
\end{array}\right.
$$

Note that

$$
R_{0}=\Gamma_{i}(0) \int_{0}^{\infty} p(a) s_{F}(a) d a+\Gamma_{e}(0) \int_{0}^{\infty}(1-p(a)) s_{F}(a) d a
$$

Then one gets (by density):

$$
\begin{aligned}
& \frac{d}{d t} \int_{0}^{\infty} \Gamma_{i}(\tau) i(t, \tau) d \tau=-\int_{0}^{\infty} \Gamma_{i}(\tau) i_{\tau}(t, \tau) d \tau-\left(\mu+\gamma_{i}\right) \int_{0}^{\infty} \Gamma_{i}(\tau) i(t, \tau) d \tau \\
& =\lambda(t) \Gamma_{i}(0) \int_{0}^{\infty} p(a) s(t, a) d a+\int_{0}^{\infty}\left[\Gamma_{i}^{\prime}(\tau)-\left(\gamma_{i}\right) \Gamma_{i}(\tau)\right] i(t, \tau) d \tau \\
& \frac{d}{d t} \int_{0}^{\infty} \Gamma_{e}(\tau) e(t, \tau) d \tau=-\int_{0}^{\infty} \Gamma_{e}(\tau) e_{\tau}(t, \tau) d \tau-\left(\mu+\gamma_{e}\right) \int_{0}^{\infty} \Gamma_{e}(\tau) e(t, \tau) d \tau \\
& =\lambda(t) \Gamma_{e}(0) \int_{0}^{\infty}(1-p(a)) s(t, a) d a+\int_{0}^{\infty}\left[\Gamma_{e}^{\prime}(\tau)-\left(\mu+\gamma_{e}\right) \Gamma_{e}(\tau)\right] e(t, \tau) d \tau
\end{aligned}
$$
so that

$\frac{d}{d t} \int_{0}^{\infty}\left[\Gamma_{i}(\tau) i(t, \tau)+\Gamma_{e}(\tau) e(t, \tau)\right] d \tau$ 
Citation: Kouakep TY, Ducroty A, Houpa DDE (2013) A Model for Hepatitis B with Chronological and Infection Ages. J Appl Computat Math 3: 148. doi:10.4172/2168-9679.1000148

Page 5 of 7

$$
\begin{aligned}
& =\left[\Gamma_{i}(0) \int_{0}^{\infty} p(a) s(t, a) d a+\Gamma_{e}(0) \int_{0}^{\infty}(1-p(a)) s_{F}(a) d a\right] \times \\
& {\left[\int_{0}^{\infty} \beta_{i}(\tau) i(t, \tau)+\beta_{e}(\tau) e(t, \tau) d \tau\right]} \\
& -\left[\int_{0}^{\infty} \beta_{i}(\tau) i(t, \tau)+\beta_{e}(\tau) e(t, \tau) d \tau\right] \\
& \frac{d}{d t} \int_{0}^{\infty}\left[\Gamma_{i}(\tau) i(t, \tau)+\Gamma_{e}(\tau) e(t, \tau)\right] d \tau
\end{aligned}
$$

so that

$$
\begin{aligned}
& \frac{d}{d t} \int_{0}^{\infty}\left[\Gamma_{i}(\tau) i(t, \tau)+\Gamma_{e}(\tau) e(t, \tau)\right] d \tau \\
& \leq\left[\Gamma_{i}(0) \int_{0}^{\infty} p(a) s(t, a) d a+\Gamma_{e}(0) \int_{0}^{\infty}(1-p(a)) s_{F}(a) d a\right] \times \\
& {\left[\int_{0}^{\infty} \beta_{i}(\tau) i(t, \tau)+\beta_{e}(\tau) e(t, \tau) d \tau\right]}
\end{aligned}
$$

Since $R_{0} \leq 1$ then,

$$
\begin{aligned}
& \frac{d}{d t} \int_{0}^{\infty}\left[\Gamma_{i}(\tau) i(t, \tau)+\Gamma_{e}(\tau) e(t, \tau)\right] d \tau \\
& \leq\left[\Gamma_{i}(0) \int_{0}^{\infty} p(a) s(t, a) d a+\Gamma_{e}(0) \int_{0}^{\infty}(1-p(a)) s_{F}(a) d a\right] \times \\
& {\left[\int_{0}^{\infty} \beta_{i}(\tau) i(t, \tau)+\beta_{e}(\tau) e(t, \tau) d \tau\right]} \\
& \leq\left[\Gamma_{i}(0) \int_{0}^{\infty} p(a) s(t, a)-s_{F}(a) d a+\Gamma_{e}(0) \int_{0}^{\infty}(1-p(a))\left[s(t, a)-s_{F}(a)\right] d a\right] \\
& \times\left[\int_{0}^{\infty} \beta_{i}(\tau) i(t, \tau)+\beta_{e}(\tau) e(t, \tau) d \tau\right]
\end{aligned}
$$

On the attractor A we check by lemma 5.1 that

$$
s(t, a) \leq s_{F}(a), \forall t \in \mathbb{R}, a>0,
$$

so that the functional

$$
V[i, e](t):=\int_{0}^{\infty}\left[\Gamma_{i}(\tau) i(t, \tau)+\Gamma_{e}(\tau) e(t, \tau)\right] d \tau,
$$

is non-increasing along the complete orbits.

$V[i, e]$ is a strict Lyapunov function for DFE on $A \subset \partial M_{0}$ and global stability of DFE when $R_{0}<1$ follows.

\section{Simulations}

We first simplify the model by assuming that $\beta_{\varepsilon}$ and $\beta_{i}$ are both constant parameters. Then introducing $I(t)=\int_{0}^{b_{0}} i(t, \tau) d \tau$ and $E(t)=\int_{0}^{\infty} e(t, \tau) d \tau$

We will use data in Tables 1-3 for the case of Cameroon.

\begin{tabular}{|l|c|c|c|}
\hline Age & prevalence $\boldsymbol{p}$ & prevalence $\boldsymbol{q}$ & Ref. \\
\hline 0 to 1 month (excluded) & 0.1 or $10 \%$ & 0.9 or $90 \%$ & {$[11,5]$} \\
\hline 1 to 6 months (included) & 0.2 or $20 \%$ & 0.8 or $80 \%$ & {$[5]$} \\
\hline 7 to 12 months (included) & 0.45 or $45 \%$ & 0.55 or $55 \%$ & {$[5]$} \\
\hline 1 to 5 years (included) & $0.5-0.25$ or $50-25 \%$ & $0.25-0.5$ or $25-50 \%$ & {$[11,5]$} \\
\hline$>5$ years & $0.94-0.9$ or $94-90 \%$ & $0.06-0.1$ or $6-10 \%$ & {$[11,5]$} \\
\hline
\end{tabular}

Table 1: "Data-(C)", some other data collected on Cameroon.

\begin{tabular}{l|c|c|c|c|c|c|}
\hline Age (years) & $\boldsymbol{p}(\mathbf{a})$ & $\boldsymbol{\beta}_{\boldsymbol{I}}$ & $\boldsymbol{B}_{E}$ & $\boldsymbol{V}_{\boldsymbol{I}}$ & $\boldsymbol{V}_{\boldsymbol{E}}$ & $\boldsymbol{\mu}$ \\
\hline$[0 ; \mathrm{A}=60 \mathrm{yrs}]$ & $0.811 . \mathrm{e}^{\left(-288^{*} \mathrm{a}\right)}$ & $10-6$ & $10-20$ & 0.036 & 0.02 & $15.10^{-5}$ \\
\hline \\
Table 2: Values for $R_{0}<1$.
\end{tabular}

\begin{tabular}{|l|c|c|c|c|c|c|}
\hline Age (years) & $\boldsymbol{p}(\mathbf{a})$ & $\boldsymbol{\beta}_{\boldsymbol{I}}$ & $\boldsymbol{B}_{E}$ & $\boldsymbol{V}_{\boldsymbol{I}}$ & $\boldsymbol{V}_{\boldsymbol{E}}$ & $\boldsymbol{\mu}$ \\
\hline$[0 ; \mathrm{A}=60 \mathrm{yrs}]$ & $0.811 . \mathrm{e}^{\left(-288^{*} \mathrm{a}\right)}$ & 0.00006 & $10-20$ & 0.09 & 0.05 & $15.10^{-5}$ \\
\hline \multicolumn{7}{c}{ Table 3: Values for $\mathrm{R}>1}$.
\end{tabular}

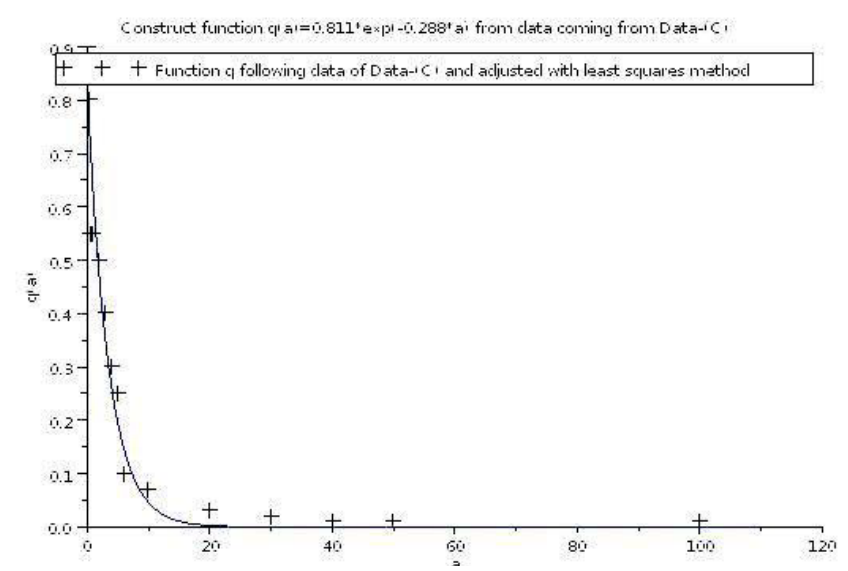

Figure 1: Construct function $q(a)=0.811^{*} \mathrm{e}\left(-0.288^{* a}\right)$ from data coming from "Data-(C)" in Table 1.

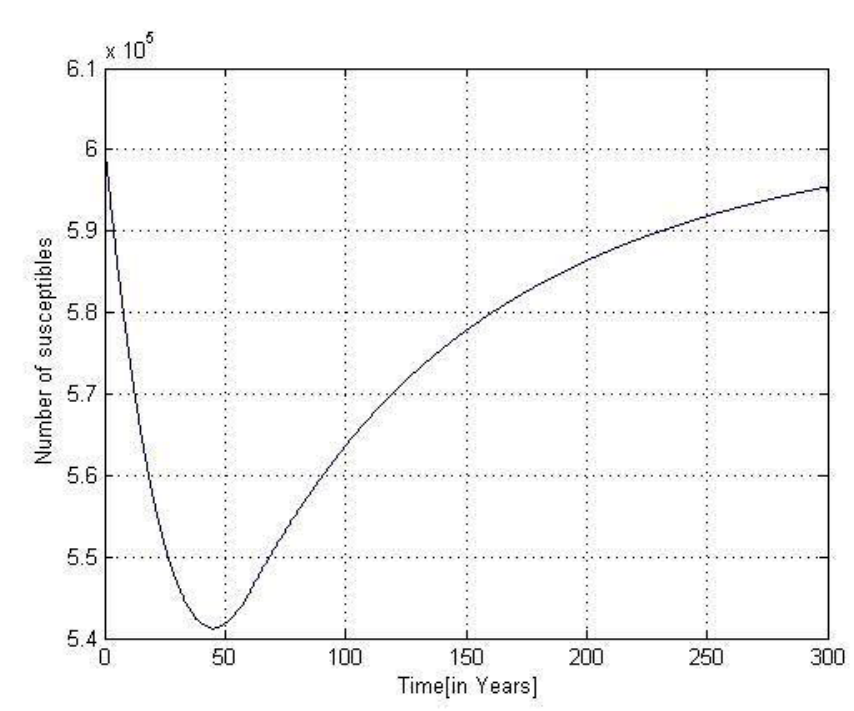

Figure 2: Function $t \rightarrow \int_{0}^{A} s(t, a) d a$ from Data-"C", $R_{0}<1$.

\section{Discussion}

Simulations illustrate the asymptotic stability of DFE in section 5. The model described by equations (1.1-1.2) exhibit a rich dynamic. We observe that the biological basic reproduction rate $R_{0}$ is fundamental for the study of the basic dynamical properties. Applied to hepatitis $B$, the model suggests that infection rates play a great role in the description of the disease (see expression of $R_{0}$ ). Simulations conducted follow our results and suggest the fact that the endemic equilibrium is asymptotically stable if $R_{0}>1$ (Figures 1-9). 


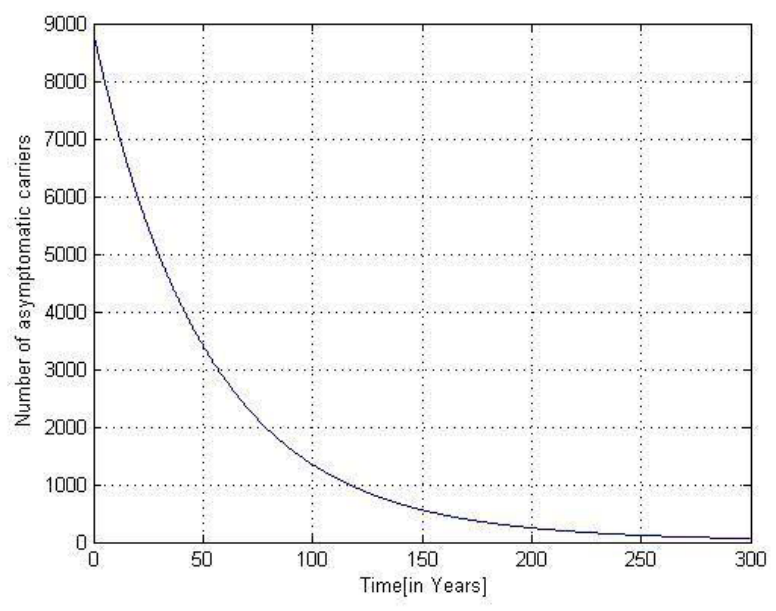

Figure 3: Function $E(t)$ from Data-"C", $R_{0}<1$

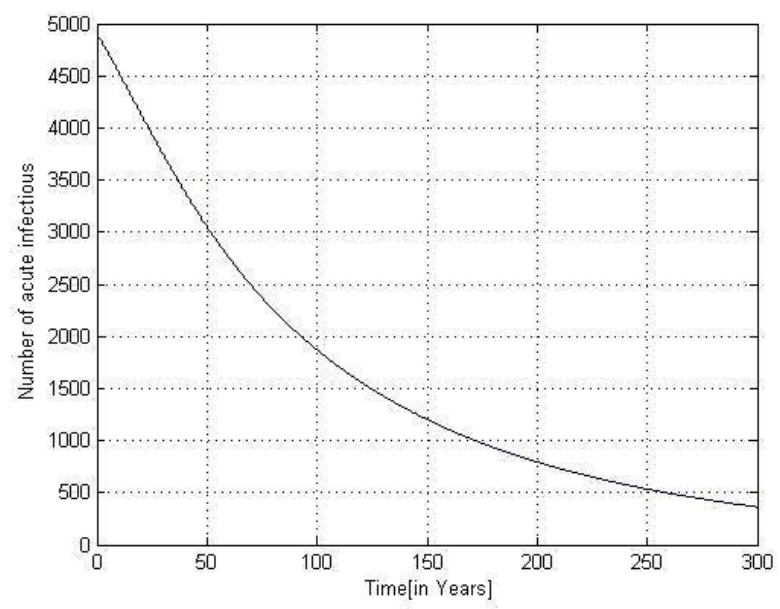

Figure 4: Function $I(t)$ from data of Data-"C", $R_{0}<1$.

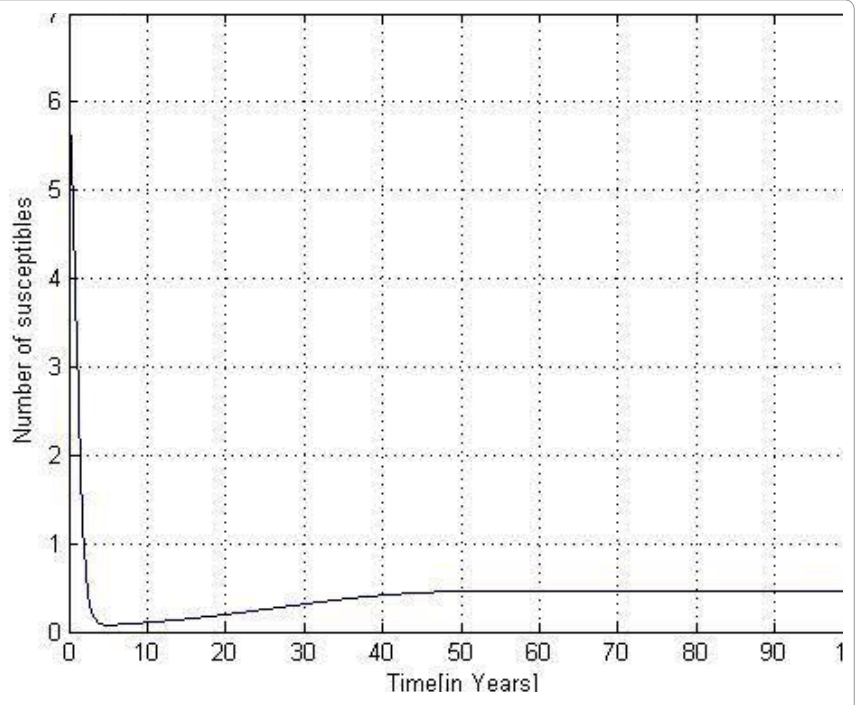

Figure 5: Function $t \rightarrow \int_{0}^{A} s(t, a) d a$ from Data-"C", $R_{0}>1$.

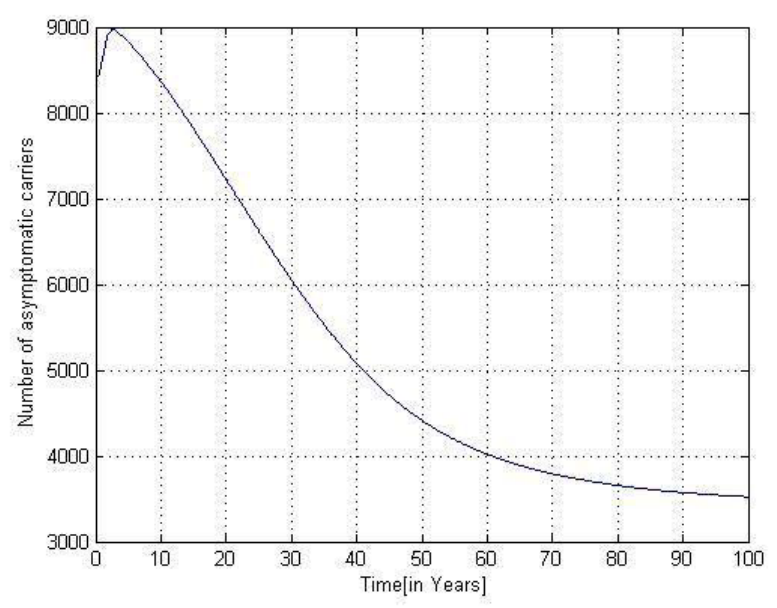

Figure 6: Function $E(t)$ from Data-"C", $R_{0}>1$.

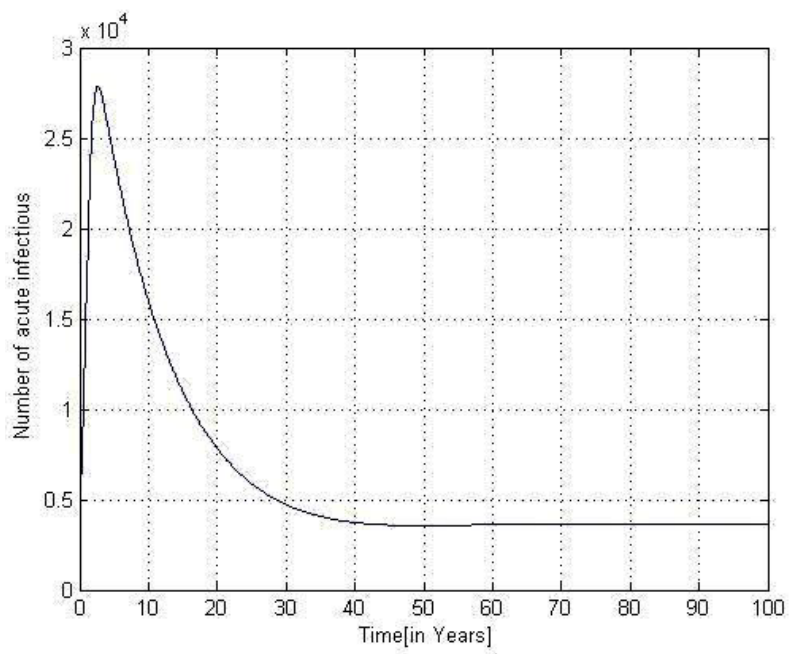

Figure 7: Function $I(t)$ from data of Data-"C", $R_{0}>1$.

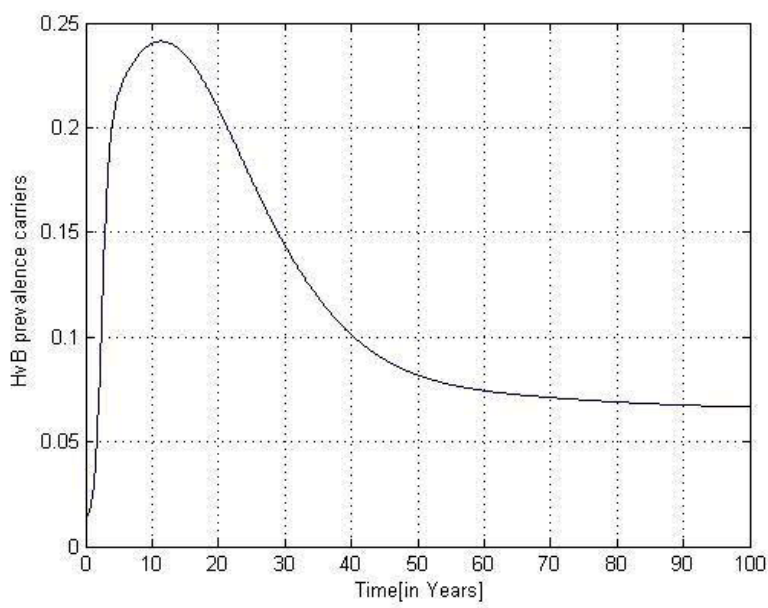

Figure 8: Prevalence of asymptomatic carriers $E(t)$ from Data-"C", $R_{0}>1$. 
Citation: Kouakep TY, Ducroty A, Houpa DDE (2013) A Model for Hepatitis B with Chronological and Infection Ages. J Appl Computat Math 3: 148. doi:10.4172/2168-9679.1000148

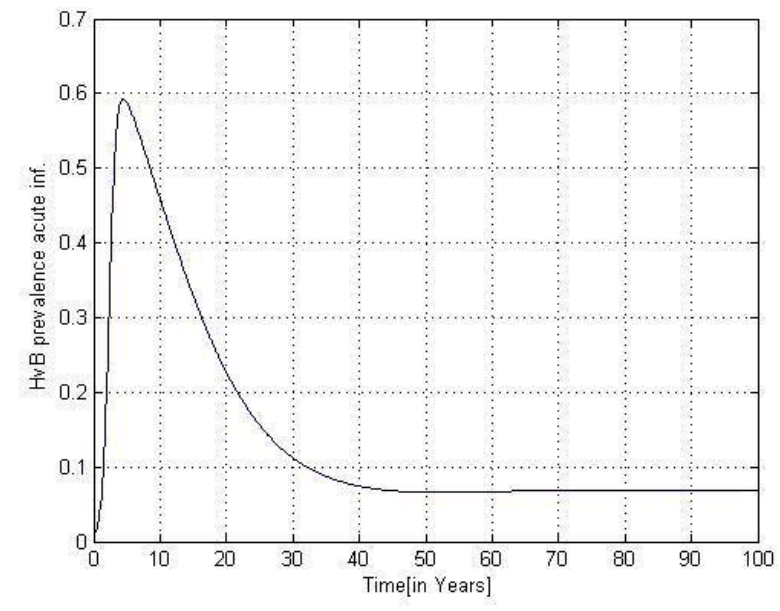

Figure 9: Prevalence of acute infectious $I(t)$ from data of Data-"C", $R_{0}>1$.

\section{References}

1. Edmunds WJ, Medley GF, Nokes DJ, O'Callaghan CJ, Whittle HC, et al.(1996). Epidemiological patterns of Hepatitis B Virus (HBV) in highly endemic areas. Epidemiology Infection 117: 313-325.

2. Momoh AA, Ibrahim MO, Madu BA (2011) Stability of Passive Immunization and Efficacy of Vaccines of Hepatitis B Model. Research Journal of Mathematics and Statistics 3: 107-110.
3. WHO (1996) Hepatitis $B$ and breastfeeding.

4. Bonzi B, Fall AA, Iggidr A, Sallet G (2010) Stability of differential susceptibility and infectivity epidemic models. J Math Biol 62: 39-64.

5. Chevret S (2010) Modeles mathematiques utilises en medicine Mathematical models in medicine. Reanimation 16: 240-244.

6. Eikenberry S, Hews S, Nagy JD, Kuang Y (2009), The Dynamics of a delay model of hepatitis B virus infection with logistic hepatocyte growth. Math Biosci Eng 6: 283-299.

7. Goldstein ST, Zhou F, Hadler SC, Bell BP, Mast EE, et al. (2005) A mathematical model to estimate global hepatitis $B$ disease burden and vaccination impact. Int J Epid 34: 1329-1339.

8. Zou L, Zhang W, Ruan S (2010) Modelling the transmission dynamics and control of Hepatitis B virus in China. J Theoret Biol 262: 330-338.

9. Zhao SJ, Xu ZY, Lu Y (2000) A mathematical model of hepatitis B virus transmission and its application for vaccination strategy in China. Int $\mathrm{J}$ Epidemiol 29: 744-752.

10. Zou L, Ruan S, Zhang W (2010) An age-structured model for trans- mission dynamics of hepatitis B. SIAM 70: 3121-3139.

11. Hethcote HW (2000) The Mathematics of Infectious Diseases. SIAM Review 42: 599-653.

12. Inaba H (1990) Threshold and stability results for an age-structured epidemic model. J Math Biol 28: 411-434.

13. Nokes DJ, Hall AJ, Edmunds WJ, Medley GF, Whittle HC (1993), The influence of age on the development of the hepatitis B carrier state. Proc Biol Sci 253: 197-201.

14. Aziz FA, Gauthier S, Abderrahman I (2008) Modelisation de la transmission verticale de l'Hepatite B. CARI.

15. Sell, George R, You, Yuncheng (2002) Dynamics of Evolutionary Equations. Springer, New York, USA. 